\title{
Time Domain Optimization using Hadamard Matrix Based Phase Sequence and DCT with Classic Frequency Domain Partition Partial Transmitted Sequence(CFDP-PTS) Scheme for the Reduction of Peak to Average Power Ratio (PAPR) in OFDM System
}

\author{
Prabal Gupta*, Balpreet Singh and Gursharanjeet Singh \\ School of ECE, Lovely Professional University, Phagwara - 144411, Punjab , India; \\ prabal.gupta9@gmail.com, balpreet.15731@|pu.co.in, gursharanjeet.singh@lpu.co.in
}

\begin{abstract}
Partial transmitted sequence (PTS) is the most important technique for the minimization of problem peak to average power ratio (PAPR) found in multicarrier system i.e., Orthogonal Frequency Division Multiplexing (OFDM). But PTS scheme undergo a very difficult and complex process of designing random phase sequences which are used for optimization of subblocks of data stream in time domain after applying Inverse Fast Fourier Transform (IFFT) so that the problem PAPR can be minimized. Hence in this paper we are proposing a technique in which we have used hadamard matrix for the optimization of sub-blocks of data stream in time domain after IFFT along with discrete cosine transform. So this proposed technique drastically reduced the complexity of designing phase sequence and help in recovering the same information back at the receiver side with the help of index of individual rows of hadamard matrix. Simulation results are presented under multiple sub carriers $(\mathrm{N})=64,128$ and 256 , classic frequency domain partition partial transmitted sequence(CFDP-PTS)=3 and 4 shows a significant gain (dB)of around 3.8 and 1.4 in comparison of conventional OFDM system and traditional PTS technique respectively under $\mathrm{N}=128$ and $\mathrm{CFDP}=4$.
\end{abstract}

Keywords: Classic Frequency Domain Partition Partial Transmitted Sequence (CFDP-PTS), Complementary Cummulative Distributive Function (CCDF), Peak to Average Power Ratio (PAPR)

\section{Introduction}

$\mathrm{OFDM}^{1}$ technique is a multicarrier transmission scheme which is used to provide very high date rate and also highly spectrally efficient technique that's why it has been adopted as the basis for $4 \mathrm{G}$, digital audio and video broadcasting. In this multicarrier transmission technique complete information is being divided into lower data stream and finally transmitted through multiple subcarriers which are orthogonal to each other hence justify the spectral efficiency of OFDM system. But apart from its advantage, it has a major draw back of a problem which known as Peak to Average Power Ratio (PAPR). The reason behind reducing this problem is that, it make the amplifier behave non-linearly and hence, distort the data in the transmitter side itself therefore several techniques have been proposed in the recent past in order to reduce this problem. Conventional PTS technique ${ }^{2,3,4,5}$ 
in which information signal is first partitioned into several sub-blocks and then passed through IFFT block so that it can be converted into corresponding time domain sequence and optimized using these random phase sequences. Finally, the sequence which generate minimum PAPR is selected for the transmission from transmitter side to receiver side and at receiver side it uses index of rows of phases sequence for the recovery of same information. Another important technique is known as Selected Mapping (SLM) $)^{6,14,15}$ in which information is optimized in frequency domain itself using multiple phase sequence of same data length which is very difficult and complex to design, and passed through IFFT at last the sequence which create minimum PAPR is selected for the transmission and same phase sequence are used at the receiver side for the recovery of same information back. Clipping $\frac{7.8}{}$ technique is used for the reduction of PAPR. This technique clips the sequence which generate maximum PAPR and hence creates distortion in the data. Coding technique uses various code like $\mathrm{BCH}$ code $\mathrm{e}^{9,10}$ in order to generate several scramblers which are added to data sequence in frequency domain and after transformation by IFFT they stored hence require very large look up tables finally, choose the sequence which generate minimum PAPR. SLM technique along with Discrete Cosine Transform (DCT) ${ }^{\frac{11,12}{12}}$ is used for the minimization of PAPR in which just before transformation by IFFT, DCT is used and each phase sequence is stored so that at last minimum PAPR sequence can be selected for the transmission. Another technique is highly optimized SLM ${ }^{13}$ in which highly optimized phases are designed in order to reduce the PAPR problem. Henceforth, in this paper we are proposing a novel technique, in which PTS is used along with hadamard phase sequence and DCT. This paper is organized as follows, Section-II presents basic definition of OFDM system, Section-III depicts proposed scheme along with block diagram, Section-IVdepicts Simulation results and finally, section- $\mathrm{V}$ shows conclusion.

\section{OFDM, PAPR, CCDF, DCT and Hadamard}

Information signal in frequency domain is being transformed into corresponding time domain using IFFT, its main aim to provide orthogonality among several subcarriers, which is shown by the following equation?"

$$
s[\mathrm{n}]=\frac{1}{\sqrt{N}} \sum_{k=0}^{N-1} S[\mathrm{k}] . \mathrm{e}^{\frac{j 2 \pi n k}{N}} \quad 0 \leq n \leq N-1
$$

Where $\mathrm{k}, \mathrm{n}$ and $\mathrm{N}$ represent frequency index, time index and number of subcarriers.

Peak to average power ratio is defined as the ratio of maximum power to the average power depicted by expectation operator is shown below [9],

$$
P A P R[\mathrm{~s}[\mathrm{n}]]=\frac{\begin{array}{c}
\max (|s[\mathrm{n}]|)^{2} \\
0 \leq n \leq N-1
\end{array}}{E\left[|s[\mathrm{n}]|^{2}\right]}
$$

Complementary Cumulative Distribution Function (CCDF) calculate the PAPR of information signal which is found to larger than specific threshold shown below ${ }^{9}$,

$$
\operatorname{CCDF}(\operatorname{PAPR}(s[n]))=\operatorname{Prob}(\operatorname{PAPR}(s[n]>\operatorname{PAPR} 0))
$$

where Prob= probability, PAPR0 is certain threshold.

Discrete Cosine Transform (DCT) 12,13 creates a sequence of some finite data as a addition of cosine function at multiple frequencies and is represented as,

$$
R(t)=\sqrt{\frac{2}{N}} \Sigma_{1=0}^{(N-1)} \beta_{1} \cdot g_{1} \cdot \cos \left(\frac{\pi t 1}{T}\right)
$$

Where $d_{1}$ is the data symbols, $\beta_{1}=0.707$ for $n=0, \beta_{1}=1$ for $\mathrm{n} \neq 0$.

Now, for $\mathrm{n}=0$ initially transformed coefficient is calculated and termed as DC co-efficients. Generally, basis functions have various waveforms which are orthogonal to one another.

Walsh hadamard matrix [16] can be generated by a methodology of selvester and rows of this matrix will be preferred for optimization in time domain for PTS technique. Henceforth, this reduces designing complexity drastically,

$$
\underset{\mathbf{H}}{\mathbf{W}}=\left[\begin{array}{cc}
\mathbf{H}_{\mathbf{N}}^{\mathbf{W}} & \mathbf{H}_{\mathbf{N}}^{\mathbf{W}} \\
\mathbf{H}_{\mathbf{N}}^{\mathbf{W}} & (-1) \mathbf{H}_{\mathbf{N}}^{\mathbf{W}}
\end{array}\right] \quad \underset{\text { and } \mathbf{H}_{\mathbf{N}}^{\mathbf{W}}}{\mathbf{W}}=\left[\begin{array}{cc}
+1 & +1 \\
+1 & -1
\end{array}\right]
$$

\section{Proposed Scheme for PAPR reduction}

Proposed scheme is detailed as under:

(i) Input data sequence of several subcarriers is defined as shown below, 


$$
\mathbf{S}=[\mathbf{S}(\mathbf{0}), \mathbf{S}(\mathbf{1}) \ldots . . . \mathrm{S}(\mathbf{N}-\mathbf{1})]
$$

Where sub-carriers $=\mathrm{N}$

(ii) Data sequence generated in equation (1) is modulated by 32 -Phase shift keying (PSK) shown below,

$$
G=\left[\mathbf{S}_{\mathbf{m}}(0), \mathbf{S}_{\mathbf{m}}(\mathbf{1}) \ldots . . . \mathrm{S}_{\mathbf{m}}(\mathbf{N}-1)\right]
$$

(iii) Above defined data sequence is partitioned in frequency domain and represented as,

$$
\begin{aligned}
& \mathbf{G}=\left[\mathbf{g}_{\mathbf{0}}, \mathbf{g}_{\mathbf{1}}, \mathbf{g}_{\mathbf{2}}, \mathbf{g}_{\mathbf{3}}, \ldots \ldots . . . \mathrm{g}_{\mathrm{D}-\mathbf{1}}\right]^{\mathrm{T}} \\
& \mathrm{G}=\sum_{\mathrm{z}=0}^{\mathrm{D}-1} \mathrm{~g}_{\mathrm{z}}
\end{aligned}
$$

where $\mathrm{D}=$ total number of partition

(iv) After partitioning information sequence is transformed using DCT shown below,

$$
R(t)=\sqrt{\frac{2}{N}} \sum_{l=0}^{(N-1)} \beta_{l} \cdot g_{\mid} \cdot \cos \left(\frac{\pi^{\star} t^{\star} I}{T}\right)
$$

(v) Now, transformed sequence is converted into time domain using IFFT is depicted as,

$$
\mathbf{r}=\operatorname{IFFT}\left[\sum_{b=0}^{D-1} f_{b} \cdot R(t)\right]
$$

(vi) Time domain optimization is performed using several rows of hadamard matrix and at last, the sequence which generate minimum PAPR value will be selected for the transmission from transmitter side to the receiver side which given by,

$$
\begin{aligned}
& \mathbf{H}_{2 \mathrm{~N}}^{\mathrm{W}}=\left[\begin{array}{cc}
\mathrm{H}_{\mathrm{N}}^{\mathrm{W}} & \mathrm{H}_{\mathrm{N}}^{\mathrm{W}} \\
\mathrm{H}_{\mathrm{N}}^{\mathrm{W}} & (-1) \mathrm{H}_{\mathrm{N}}^{\mathrm{W}}
\end{array}\right] \text { and } \quad \mathbf{H}_{\mathrm{N}}^{\mathrm{W}}=\left[\begin{array}{cc}
+1 & +1 \\
+1 & -1
\end{array}\right] \\
& \mathrm{y}=\sum_{\mathrm{d}=0}^{\mathrm{D}-1} \mathrm{f}_{\mathrm{d}} \cdot \operatorname{ll} \mathrm{IPI}[\mathrm{R}(\mathrm{t})] \quad \text { and } \quad \mathrm{y}=\sum_{\mathrm{d}=0}^{\mathrm{D}-1} \mathrm{f}_{\mathrm{d}} \cdot \mathrm{r}_{\mathrm{d}}
\end{aligned}
$$

Where $\mathrm{fd}=$ rows of phase sequence selected from above defined matrix

\section{Simulations, Results and Discussion}

Simulations results are generated and presented for proposed scheme in comparison with conventional OFDM system and PTS where number of subcarriers $(\mathrm{N})$ are
$=64,128$ and 256 , oversampling factor $=4$, total number of OFDM symbol $=10000$ and modulation scheme $=32-$ PSK.

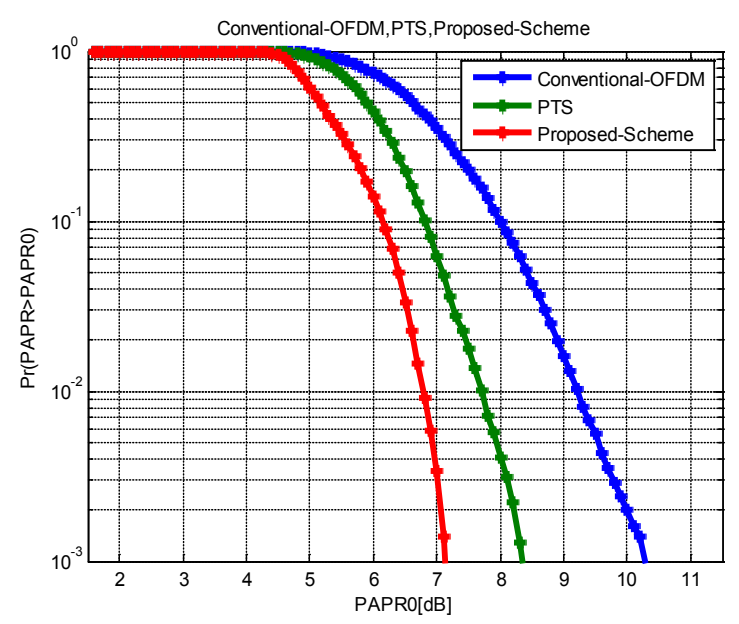

Figure 1. Comparative analysis of conventional OFDM system, PTS,Proposed scheme under $\mathrm{N}=64$, partition=3, oversampling $=4$.

In the above Figure 1, results are presented at $\mathrm{CCDF}=10^{-3}$ depicts PAPR in $\mathrm{dB}$ for Conventional OFDM, PTS and Proposed Scheme at N=64, Number of partition $=3$, over sampling $=4$, modulation scheme $=32$-PSK are 10.2, 8.3, 7.1 respectively. Henceforth, it can inferred that minimum PAPR value is obtained by our proposed scheme which shows the remarkable performance of our proposed scheme.

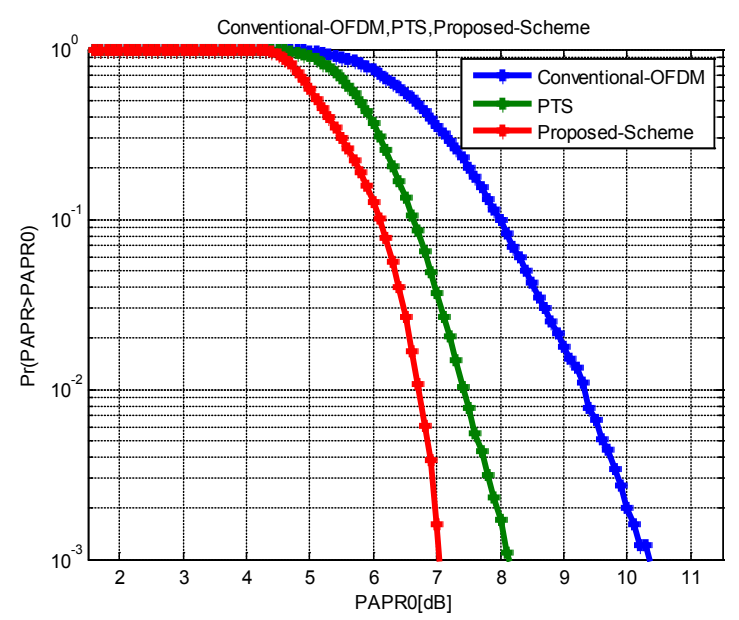

Figure 2. Comparative analysis of conventional OFDM system, PTS,Proposed scheme under N=64, partition=4, oversampling $=4$.

In the above Figure 2, results are presented at $C C D F=10-3$ depicts PAPR in $\mathrm{dB}$ for Conventional OFDM, PTS and 
Proposed Scheme at $\mathrm{N}=64$, Number of partition=4, over sampling $=4$, modulation scheme $=32$-PSK are $10.3,8.1$, 7.0 respectively. Henceforth, it can inferred that minimum PAPR value is obtained by our proposed scheme which shows the remarkable performance of our proposed scheme.

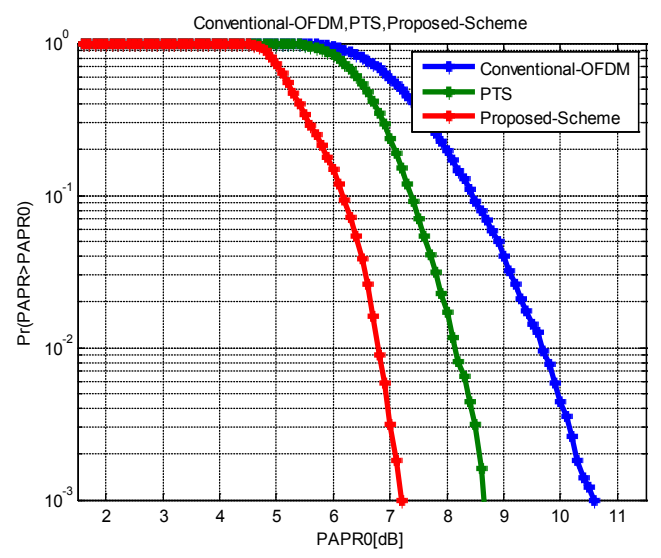

Figure 3. Comparative analysis of conventional OFDM system, PTS,Proposed scheme under $\mathrm{N}=128$, partition=3, oversampling $=4$.

In the above Figure 3, results are presented at $\mathrm{CCDF}=10-3$ depicts PAPR in $\mathrm{dB}$ for Conventional OFDM, PTS and Proposed Scheme at N=128, Number of partition $=3$, over sampling $=4$, modulation scheme $=32$ PSK are 10.6, 8.6, 7.2 respectively. Henceforth, it can inferred that minimum PAPR value is obtained by our proposed scheme which shows the remarkable performance of our proposed scheme.

In the above Figure 5, results are presented at $\mathrm{CCDF}=10-3$ depicts PAPR in $\mathrm{dB}$ for Conventional OFDM, PTS and Proposed Scheme at N=128, Number of partition $=4$, over sampling $=4$, modulation scheme $=32$-PSK are 10.9, 8.5, 7.1 respectively. Henceforth, it can inferred that minimum PAPR value is obtained by our proposed scheme which shows the remarkable performance of our proposed scheme.

In the above Figure 6, results are presented at $\mathrm{CCDF}=10-3$ depicts PAPR in $\mathrm{dB}$ for Conventional OFDM, PTS and Proposed Scheme at $\mathrm{N}=256$, Number of partition $=3$, over sampling $=4$, modulation scheme $=32$-PSK are 10.8, 9.3, 7.1 respectively. Henceforth, it can inferred that minimum PAPR value is obtained by our proposed scheme which shows the remarkable performance of our proposed scheme.

In the above Figure 7, results are presented at $\mathrm{CCDF}=10-3$ depicts PAPR in $\mathrm{dB}$ for Conventional OFDM, PTS and
Proposed Scheme at $\mathrm{N}=256$, Number of partition $=4$, over sampling $=4$, modulation scheme $=32$-PSK are 10.8, 8.9, 7.1 respectively. Henceforth, it can inferred that minimum PAPR value is obtained by our proposed scheme which shows the remarkable performance of our proposed scheme.

$$
\mathbf{d}_{\text {optimz }}=\arg \min \left(\operatorname{PAPR}\left(\mathbf{d}^{\mathbf{n}}\right)\right)
$$

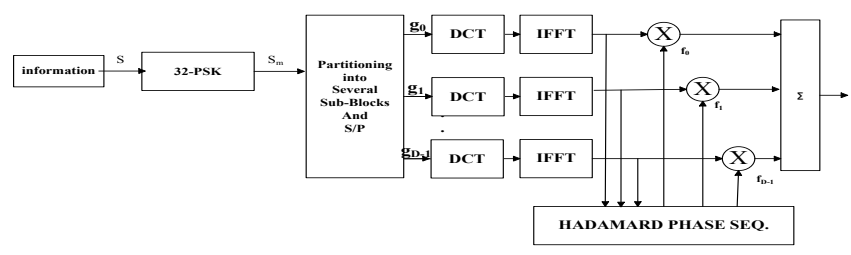

Figure 4. Block diagram of proposed scheme.

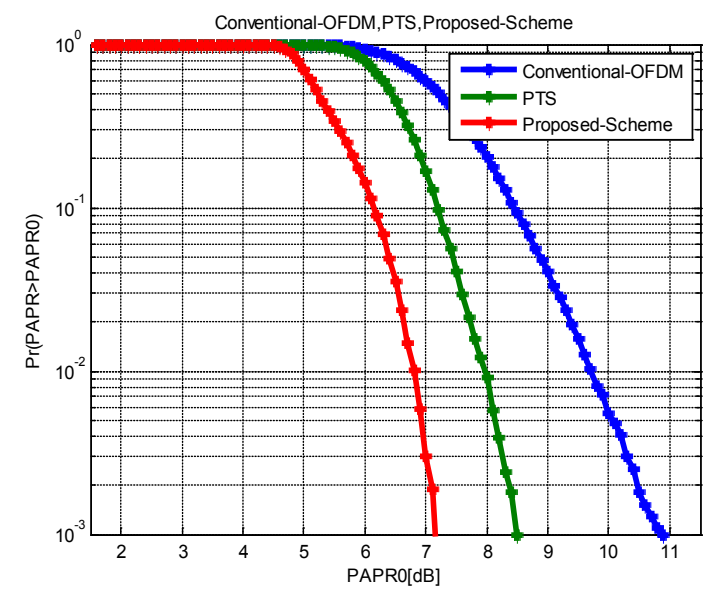

Figure 5. Comparative analysis of conventional OFDM system, PTS,Proposed scheme under $\mathrm{N}=128$, partition $=4$, oversampling $=4$.

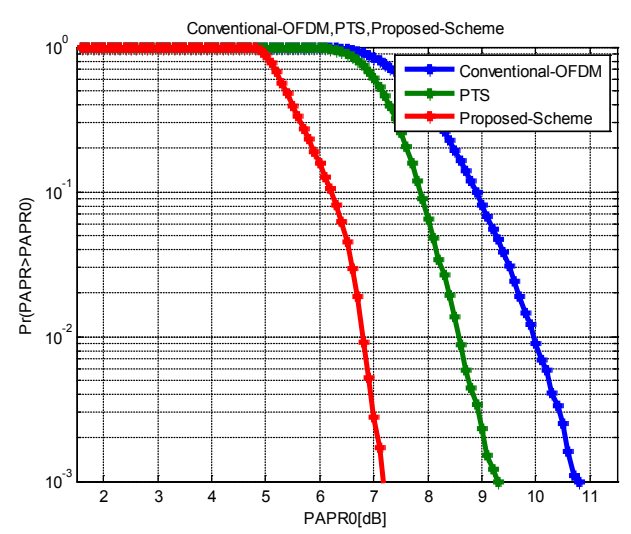

Figure 6. Comparative analysis of conventional OFDM system, PTS,Proposed scheme under $\mathrm{N}=256$, partition $=3$, oversampling $=4$. 


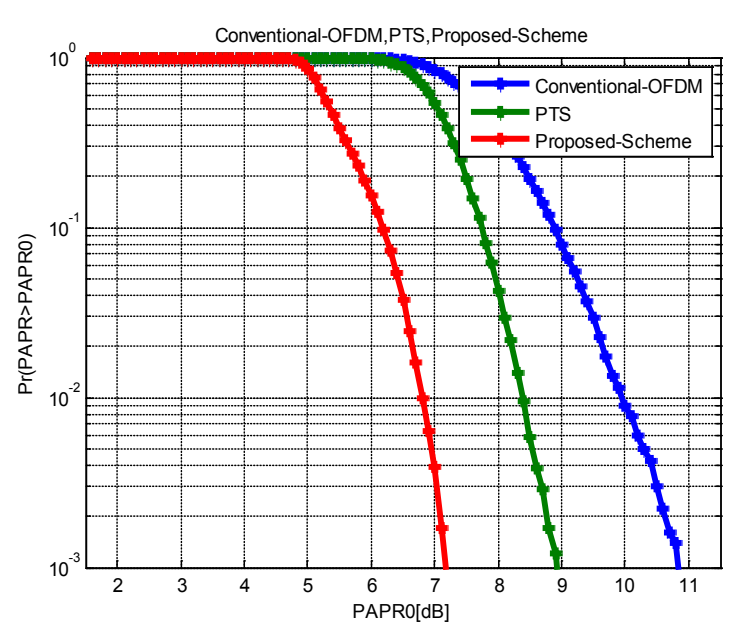

Figure 7. Comparative analysis of conventional OFDM system, PTS,Proposed scheme under $\mathrm{N}=256$, partition=3, oversampling $=4$.

Table 1. Performance Evaluation of Proposed Scheme with Conventional Ofdm System And Pts at $\mathbf{C C D F}=\mathbf{1 0}^{-3}$

\begin{tabular}{|l|c|c|c|c|c|c|}
\hline Parameters & $\mathbf{6 4 - 3}$ & $\mathbf{6 4 - 4}$ & $\mathbf{1 2 8 - 3}$ & $\mathbf{1 2 8 - 4}$ & $\mathbf{2 5 6 - 3}$ & $\mathbf{2 5 6 - 4}$ \\
\hline $\begin{array}{l}\text { Conventional } \\
\text { OFDM }\end{array}$ & 10.2 & 10.3 & 10.6 & 10.9 & 10.8 & 10.8 \\
\hline PTS & 8,3 & 8.1 & 8.6 & 8.5 & 9.3 & 8.9 \\
\hline $\begin{array}{l}\text { Proposed } \\
\text { Scheme }\end{array}$ & 7.1 & 7 & 7.2 & 7.1 & 7.1 & 7.1 \\
\hline
\end{tabular}

Table 2. Gain Achieved By Our Proposed Scheme as Compared With Conventional Ofdm System and Pts

\begin{tabular}{|l|c|c|c|c|c|c|}
\hline Parameters & $\mathbf{6 4 - 3}$ & $\mathbf{6 4 - 4}$ & $\mathbf{1 2 8 - 3}$ & $\mathbf{1 2 8 - 4}$ & $\mathbf{2 5 6 - 3}$ & $\mathbf{2 5 6 - 4}$ \\
\hline $\begin{array}{l}\text { Conventional } \\
\text { OFDM }\end{array}$ & 3.1 & 3.3 & 3.4 & 3.8 & 3.7 & 3.7 \\
\hline PTS & 1.7 & 1.1 & 1.4 & 1.4 & 2.2 & 1.8 \\
\hline
\end{tabular}

\section{Conclusion}

Classic frequency domain partition partial transmitted sequence(CFDP-PTS) scheme is proposed in this paper for the reduction of peak to average power ratio in OFDM system. In this work we have preferred $\mathrm{N}=64,128$ and 256, frequency domain partition=3 and 4 with oversampling factor $=4$. Since the conventional scheme suffer in designing appropriate phase sequence so our prop osed work do it very efficiently and at the same time in each and every case presented in simulation and results section clearly show the gain, efficiency and remarkable performance of our proposed work for the minimization of PAPR problem.

\section{Acknowledgement}

Prabal Gupta, is very thankful to Mr. Shivguru, Ms. Durgbhama, Ms. Durgnashini, for their worthy observations and guidance in order to improve the quality of this work.

\section{References}

1. Tao. Jiang, Yiyan.Wu. An overview Peak-to-Average Power Ratio Reduction Techniques for OFDM Signals. Broadcasting. IEEE Trans. June 2008; 54(2): 257-68.

2. Tellambura C.Improved phase factor computation for the PAR reduction of an OFDM signal using PTS”, IEEE Communications Letters . 2001; 5(4):135-37.

3. Sathananthan K, Tellambura C. Partial transmit sequence arid selected mapping schemes to reduce ICI in OFDM systems., IEEE Communications Letters. 2002; 6(8):313-15.

4. Oh-Ju Kwon, Yeong-Ho Ha.Multi-carrier PAP reduction method using sub-optimal PTS with threshold. IEEE Transactions on Broadcasting. 2003; 49(2):232-36.

5. Young-Hwan You, Won-Gi Jeon, Jong-Ho Paik, Hyeok-Koo Jung.Low-complexity PAR reduction schemes using SLM and PTS approaches for OFDM-CDMA signals., IEEE Transactions on Consumer Electronics. 2003; 49(2):284-89.

6. Woo. Jun-Young, Joo. Hyun Seung, Kim. Kee-Hoon, No. Jong-Seon, Shin. Dong-Joon. PAPR Analysis of ClassIII SLM Scheme Based on Variance of Correlation of Alternative OFDM Signal Sequences. IEEE Communication Letters. 2015; 19(6): 989-92.

7. Sharma C., Sharma PK., Tomar SK., Gupta AK.A modified Iterative Amplitude clipping and filtering technique for PAPR reduction in OFDM systems. Proc. Conf. ETNCC2011.April 2011;p. 365-68.

8. Deepa T, Swetha K, Kumar R. A joint clipping and logarithmic based companding for the reduction of peakto-average power ratio in OFDM system. Proc. IEEE Conf. ICICES-2013. Feb 2013. p. 655-59.

9. Gupta P, Kumar BA, Jain SK. Peak to average power ratio reduction in OFDM using higher order partitioned PTS sequence and Bose Chaudhuri Hocquenghem Codes. Proc. IEEE Conf. SPACES-2015. Jan 2015. p. 443-47.

10. Li L, Qu D.Jiang T.Partition optimization in LDPC-Coded OFDM systems with PTS PAPR reduction.Vehicular Technology. IEEE Trans. Oct 2014. p. 4108-113.

11. Prabal Gupta, Singh RK. A hybridized discrete cosine transform based peak to average power ratio reduction in OFDM system using suboptimal Qth circular shifting 
phase sequence generated matrix (QSCPM) for selected mapping. 2016 International Conference on Computer Communication and Informatics. P. 1-5.

12. Jeoti Baig V. DCT precoded SLM technique for PAPR reduction in OFDM systems. Proc IEEE Conf. ICIAS-2010. June 2010. p. 1-6.

13. Prabal Gupta, Singh RK.Highly optimized Selected Mapping based peak to average power ratio reduction OFDM system using different modulation schemes., Third International Conference on Image Information Processing (ICIIP). 2015. p. 261-64.

14. Hill. G, Faulkner. M, Singh. J.Cyclic shifting and time inversion of partial transmit sequence to reduce the peak to average power ratio in OFDM. IEEE. 2000. p. 1256-59.

15. Hill. G, Faulkner. M, Singh.Reducing the peak to average power ratio in OFDM by cyclically shifting partial transmitted sequence.2000; 36:561.

16. Deepa T, Swetha K, Kumar R. A joint clipping and logarithmic based companding for the reduction of peakto-average power ratio in OFDM system. In Proc. IEEE Conf. ICICES-2013. Feb. 2013. p. 655-659.

17. Peng C, Yue X, Dan Lilin, Li Shaoqian. Optimized phase sequence set for SLM-OFDM Communications. Proc. Conf. ICCCAS. 2007. p. 284-87. 